durch informelles Verwaltungshandeln unter "B. I. 2. b.) bb.) (2) (b) (aa) (bbb)" erscheint! Einfacher wäre: S. 145. Schließlich werden für das Inhaltsverzeichnis vor dem Text und das Literaturverzeichnis nach dem Text die gleichen römischen Ziffern als Seitenzahlen verwendet.

Karl Leuteritz

Daniel Thürer/Stephan Kux (Hrsg.)

GATT 1994 und die Welthandelsorganisation - Herausforderung für die Schweiz und Europa

Schulthess Polygraphischer Verlag, Zürich / Baden-Baden, 1996, 344 S., SFr 82.00

Noch vor der Errichtung der World Trade Organization zum 1.1.1995 veranstalteten zwei schweizerische Europa-Institute eine gemeinsame Tagung, um "die neue Welthandelsorganisation (WTO) schweizerischen und ausländischen Fachleuten aus Politik, Wirtschaft, Verwaltung, Medien und Verbänden näherzubringen und den Dialog zwischen Wissenschaft und Praxis zu fördern", also "einen nützlich Überblick über das umfassende Regelwerk der neuen Weltwirtschaftsorganisation zu geben" (so Vischer / Schluep, Vorwort). Neben der "Einleitung" - die zugleich eine Zusammenfassung der einzelnen Referate enthält - und "Schlußfolgerungen" der Herausgeber umfaßt der Tagungsband 20 Beiträge, in denen mehr oder weniger detailliert (und nicht immer mit wissenschaftlichem Apparat versehen) dem eingangs erhobenen Anspruch Genüge getan wird, wobei ein sorgfältiges Stichwortverzeichnis gute Unterstützung leistet. So findet man dort nicht nur allgemeine Verweisungen z.B. auf "Entwicklungsländer", sondern unter diesem Stichwort Hinweise etwa auf die Haltung(en) dieser Staaten zu Fragen des geistigen Eigentums, zu Umweltschutzund Sozialklauseln oder auch zu Zollproblemen.

Die Qualität der einzelnen Beiträge ist überaus unterschiedlich: Einige erschöpfen sich in einem Aufriß des Themas (Zeller zur WTO) und/oder dienen primär zu wirtschaftspolitischen Statements (Blankart); andere manifestieren allein die Sicht eines einzelnen Wirtschaftszweiges (Schwietert, Becker), teils freilich mit beachtlichem Tiefgang (Horber zur Landwirtschaft). Zur Thematik eines "Sozialdumping durch Liberalisierung des Welthandels" wird nicht nur aus gewerkschaftlicher (Baumann) wie aus universitärer Sicht (Emmert), sondern auch mehrfach in einem breiteren Kontext Stellung bezogen (z.B. Benedek, S. 34; Thürer, S. 67, 70 f.).

Benedek kommt in seiner "Einführung in die Ergebnisse der Uruguay-Runde" zu dem Schluß, hier habe "die bedeutendste Vertiefung und Erweiterung des multilateralen Handelssystems in seiner Geschichte" stattgefunden; zudem stehe nunmehr "auch im Bereich des internationalen Handels eine vollwertige internationale Organisation für die Unterstützung der internationalen Zusammenarbeit zur Verfügung" (S. 32). Thürer fügt dem hinzu, 
in dem "durch das WTO-Übereinkommen den zuständigen Organen erteilten Auftrag, organisatorische Verbindungen zu Weltbankgruppe und Währungsfonds zu knüpfen" (Art. III Abs. 5), werden nun auch "der Gedanke der Einheit der internationalen Wirtschaftsverfassung als Teil der Verfassungsordnung der internationalen Gemeinschaft als ganzer wieder deutlich hervorgehoben" (S. 46); ähnlich äußert sich Tran Van-Thinh (S. 77). Allerdings bleibt der nunmehr normierte Stand immer noch hinter den Regeln der Havanna-Charta über eine I(nternational) T(rade) O(rganization) von 1947/48 zurück (S. 97 f.), so daß Schluep als ein "zentrales Nicht-Ergebnis" der Uruguay-Runde "das klare und uneingeschränkte Bekenntnis zum Gehirn der Marktwirtschaft, zur Garantie des wirksamen Wettbewerbs" konstatiert (S. 96). Unterschiedlich wird die neue, striktere Ausgestaltung der Streitbeilegung bewertet; insbesondere Hauser sieht im "quasi-gerichtlichen Verfahren" auch Probleme (S. 90 f.).

Dem G(eneral) A(greement on) T(rade in) S(ervices) sind vier Aufsätze gewidmet; hauptsächliches Augenmerk wird dabei vor allem Finanz-, aber auch TelekommunikationsDienstleistungen (Senti / Weber, S. 146, 153 f.) geschenkt, bei denen der Liberalisierungsprozeß nach wie vor überaus zäh verläuft. Kakabadse und Kampf vermerken hierbei den engen Zusammenhang zwischen Dienstleistungsfreiheit und Investitionspolitik (S. 162, 179); letzterer übt zudem Kritik an der EuGH-Entscheidung (Rs. 1/94) zur Kompetenzverteilung zwischen EG und Mitgliedstaaten (S. 184). Während T(rade-) R(elated) I(nvestment) M(easure)s nur nebenbei erwähnt sind (vor allem bei Benedek), bildet das Abkommen über handelsrelevante Aspekte an geistigem Eigentum (TRIPS) das Thema zweier Aufsätze (Cottier, Becker); beide unterstreichen die Bedeutung gerade für "newly industrializing countries", aber auch für andere Entwicklungsländer.

Auf der Agenda der WTO werden weiterhin die Reizworte "Öko-" und "Sozialdumping" stehen. Mehrere Autoren teilen diesbezüglich die Auffassung, es handele sich hierbei um wichtige Fragen der internationalen (Wirtschafts-)Politik. Kux sieht insoweit bereits heute eine wichtige Aufgabe des verbesserten "dispute settlement" (S. 290), während Baumann den Zusammenhang mit der internationalen Migration (Arbeitnehmer-Freizügigkeit) verdeutlicht (S. 297 f., 301 f.). In einem profunden, teils provokativen Beitrag - "There is no such Thing as a free Lunch!" - kommt Emmert zum Ergebnis, die im Interesse der gesamten Menschheit gebotene Einhaltung weltweit höherer Umwelt- (und Sozial-) Standards solle auf neuen, kreativen Wegen - auch handelsbezogen - verwirklicht werden; besser als Sanktionen seien (finanzielle) Anreize für Entwicklungsländer und verbindliche Vorgaben gegenüber "multinationalen" Unternehmen (S. 325). Thürer / Kux sprechen sich (auch insoweit?) für eine "Privatisierung staatlicher Außenwirtschaftspolitik" aus (S. 329), wobei jedoch neben der "Garantie der privaten Wettbewerbsfreiheit" zugleich "eine auch gegen Private gerichtete Ordnungspolitik erforderlich " sei. Die WTO müsse ein "offenes, durchlässiges Interaktionssystem" bilden. Dessen Konturen deuten die Herausgeber freilich nur vage an, mit dem Hinweis auf die "Vielschichtigkeit der Wirtschaftsordnung, die weder rein national noch vollständig global, weder rein staatlich noch vollständig privat ist, sondern eine komplexe Mischform bildet mit nationalen und globalen, staatlichen und privaten 
Elementen, multilateral, plurilateral und transnational organisiert, hierarchisch und vernetzt" (S. 329). Zur Auflösung dieser "confusion at a higher level" bietet der Band reichhaltiges Material, auf dem weiter gebaut werden kann und muß, um eines "global sustainable development" willen.

Ludwig Gramlich

\section{Andreas Diem}

Freihandel und Umweltschutz in GATT und WTO

Nomos Verlagsgesellschaft, Baden-Baden, 1996, 210 S., DM 65,--

Die als Dissertation in Tübingen von Thomas Oppermann betreute Arbeit verdankt ihre Entstehung, wie aus dem Vorwort hervorgeht (S. 5), der höchst kontrovers diskutierten Entscheidung eines GATT Panel aus dem Jahr 1991 ("Tuna I"), in welchem einseitige Importbeschränkungen der USA im Hinblick auf Thunfisch(produkte) für unvereinbar mit dem GATT (1947) erachtet wurden, obgleich diese Maßnahmen primär darauf abzielten, gegen Fangmethoden (mexikanischer Fischer) auf Hoher See anzugehen, bei denen auch Delphine in großer Zahl getötet wurden (näher S. 33 ff.). Damit reiht sie sich in die Diskussion ein, die vor allem im Vorfeld der UNCED 1992 über ein "Greening of the GATT" begann und nach der Errichtung der Welthandelsorganisation (WTO) zum 1.1.1995 auch in deren Rahmen, vor allem im Committee on Trade and Environment, vertieft fortgeführt wird. Freilich kam bis zur ersten Ministerkonferenz (Singapur, Dezember 1996) zwar ein ausführlicher Arbeitsbericht zustande; greifbare Ergebnisse wurden jedoch nicht erreicht. Andererseits weckt das erste nach den neuen WTO-Regeln abgeschlossene Streitbeilegungsverfahren (über die Vereinbarkeit von auf den U.S. Clean Air Act gestützten Handelshemmnissen insbesondere mit dem GATT 1994 s. S. 58 f.) durchaus Erwartungen, daß in diesem Rahmen zumindest weithin akzeptable Einzelfall-Lösungen zustanden kommen können. Deren Umsetzung durch unterlegene Staaten ist damit jedoch noch nicht gesichert; $\mathrm{da}$ die Vereinigten Staaten hier die Beachtung der WTO-Entscheidung zugesagt haben (s. WTO Focus no. 14 [1996], S. 2), sollte aber ein gutes Omen sein.

Diem gibt in seiner Studie, die das neue WTO-Recht noch erfassen konnte (S. $52 \mathrm{ff}$.) - und die wichtigsten umweltbezogenen Regeln im Anhang wiedergibt -, andererseits das Thema "Ökodumping" (weithin) ausspart (S. 15), zunächst einen geschichtlichen Überblick auf das Wechselspiel von Freihandel und Umwelt (B.). Mehrfach weist er darauf hin, daß bereits 1911 ein multilateraler Vertrag die Handels-Aspekte umweltgefährdender (Phosphor-)Produktion einbezog (S. 18 f., 130 f.). Ein besonderes Augenmerk gilt in Teil B. den seit 1982 abgegebenen Panel-Berichten zum Umweltbereich (S. 24 ff.), die jedoch auch später immer wieder eingearbeitet werden. 